Trans

continentales

\title{
Transcontinentales
}

Sociétés, idéologies, système mondial

Afrique plurielle

\section{Iran, une puissance régionale sans expérience}

Iran: An Inexperienced Regional Power?

\section{Bernard Hourcade}

\section{(2) OpenEdition}

\section{Journals}

Édition électronique

URL : http://journals.openedition.org/transcontinentales/472

DOI : 10.4000/transcontinentales.472

ISBN : 978-2-8218-1408-0

ISSN : 1775-397X

\section{Éditeur}

Editions de la maison des sciences de l'homme

\section{Édition imprimée}

Date de publication : 30 juin 2006

Pagination : 109-120

ISBN : 2200-92169-1

ISSN : 1950-1684

\section{Référence électronique}

Bernard Hourcade, «Iran, une puissance régionale sans expérience », Transcontinentales [En ligne], 2 | 2006, document 8, mis en ligne le 30 septembre 2012, consulté le 08 septembre 2020. URL : http:// journals.openedition.org/transcontinentales/472 ; DOI : https://doi.org/10.4000/transcontinentales. 472

Ce document a été généré automatiquement le 8 septembre 2020

Tous droits réservés 


\title{
Iran, une puissance régionale sans expérience
}

\author{
Iran: An Inexperienced Regional Power?
}

\author{
Bernard Hourcade
}

$1 \mathrm{Au} \mathrm{XIX}^{\mathrm{e}}$ siècle, le royaume de Perse n'avait aucun intérêt. C'était tout juste un no man's land entre les empires ottoman, russe et britannique, un territoire qui ne valait même pas une guerre coloniale. Contrairement à une relecture nationaliste iranienne de l'histoire, les rivalités anglo-russes qui ont émaillé la vie politique de la Perse étaient mineures en comparaison des enjeux coloniaux en Inde, en Afrique ou en Asie centrale. Tout a changé après 1908, avec la découverte du pétrole qui a permis à l'ancienne Perse de s'intégrer dans la vie économique et politique du $\mathrm{xx}^{\mathrm{e}}$ siècle. En un siècle, l'Iran moderne s'est forgé une nouvelle identité. Il veut être reconnu comme une nouvelle puissance régionale ${ }^{1}$. Cette transformation rapide ne s'est pas passée sans drame et reste encore inachevée, si bien que la République islamique d'Iran n'est pas toujours considérée comme partenaire potentiel, en raison de son idéologie, mais aussi de son manque d'expérience et donc de fiabilité dans son nouveau rôle international.

2 Quand on parle de l'Iran, il est de bon ton d'introduire son propos par des compliments, parfois flagorneurs, sur la gloire des empires antiques, sur les beautés d'Ispahan, la subtilité des poèmes de Hâfez ou la continuité d'une civilisation qui a su préserver et reconstruire son identité pendant plus de trois millénaires. Viennent ensuite des propos souvent sévères sur le comportement de ce pays longtemps marginal, paradoxal et incontrôlable, devenu au cours du siècle passé le second pays exportateur de pétrole, le ré-inventeur de l'islam politique, le premier « rival » des États-Unis, et une puissance régionale dont les ambitions nucléaires sont fortes, affichées et avérées. Les références au passé prestigieux sont parfois une façon implicite de ne pas prendre l'Iran actuel au sérieux ou d'occulter la nouvelle réalité des rapports de force.

3 Par le passé, cette ignorance volontaire a conduit à de graves erreurs d'analyse : en se fondant sur le mythe de la continuité impériale millénaire, on a affirmé en 1978 que le Chah était inamovible; en prenant les religieux chiites pour des érudits médiévaux, on a découvert trop tard la force de l'islam moderne ; et en confinant la culture persane à 
la poésie, on a oublié la qualité des scientifiques iraniens et leur capacité à maîtriser l'énergie nucléaire. Cette situation impose de réviser les modèles d'analyse, notamment la dichotomie entre «tradition » et «modernité » qui ne permet plus de comprendre les dynamiques actuelles d'un pays dont le problème majeur est peut-être moins l'ambition nationaliste ou l'idéologie islamiste, que les ambitions de la jeunesse, l'isolement et le manque d'expérience internationale pour appréhender et contrôler sa propre complexité sociale, culturelle et politique.

Depuis les premiers moments de la Révolution islamique, l'originalité de la recomposition des identités et des dynamiques sociales a été mise en évidence par plusieurs chercheurs ${ }^{2}$, mais la vivacité du mythe de l'empire Achéménide et de Darius est restée la plus forte. Par ailleurs, certains aspects de l'islam politique, le voile des femmes ou le gouvernement des religieux (mollahs) par exemple, occultaient la montée irréversible de forces nouvelles comme la socialisation des femmes ${ }^{3}$. Depuis 2003, la crise du nucléaire iranien impose un retour à la réalité qui pourrait être salutaire si la communauté internationale prend l'Iran au sérieux et surtout si l'ancienne Perse prend conscience de ses nouvelles responsabilités.

\section{Islam, Iran, International : les trois composantes de l'identité iranienne}

5 On ne peut plus comprendre l'Iran - et probablement bien des pays émergents - en opposant simplement, comme au XIX ${ }^{\mathrm{e}}$ siècle, «tradition » et "modernité ». Ce modèle d'analyse rudimentaire pouvait être utile quand le pays avait peu de contacts avec le monde industrialisé, mais ce n'est plus le cas de l'Iran depuis longtemps. On a souvent rangé l'islam dans la «tradition». Pourtant, on ne peut que constater que les « mollahs » iraniens forment désormais le clergé le plus « moderne » du monde grâce à l'expérience de trois décennies de gestion d'un État complexe. Il convient en outre de rappeler que c'est en 1979, date de la Révolution islamique, que la population iranienne est devenue en majorité citadine et alphabétisée, résultat de la Révolution blanche du Chah en $19622^{4}$. Cela ne signifie pas que la chute de l'empire ait été mécaniquement provoquée par un changement social, mais la coöncidence ne saurait être ignorée.

6 L'identité et la dynamique socio-politique et culturelle de l'Iran sont désormais structurées autour de trois composantes principales - les trois « $\mathrm{i} »$ : Islam, Iran, et International. Ces trois forces complexes, concurrentes mais complémentaires, se sont construites tout au long $\mathrm{du} \mathrm{xx}^{\mathrm{e}}$ siècle et sont devenues incontournables : l'islam autour du chiisme et de l'islam politique, l'Iran autour de la diversité ethno-culturelle, du nationalisme et du changement social, l'international autour de la science, des universités et surtout du pétrole dont les exportations lient l'ancienne Perse au reste du monde ${ }^{5}$. 


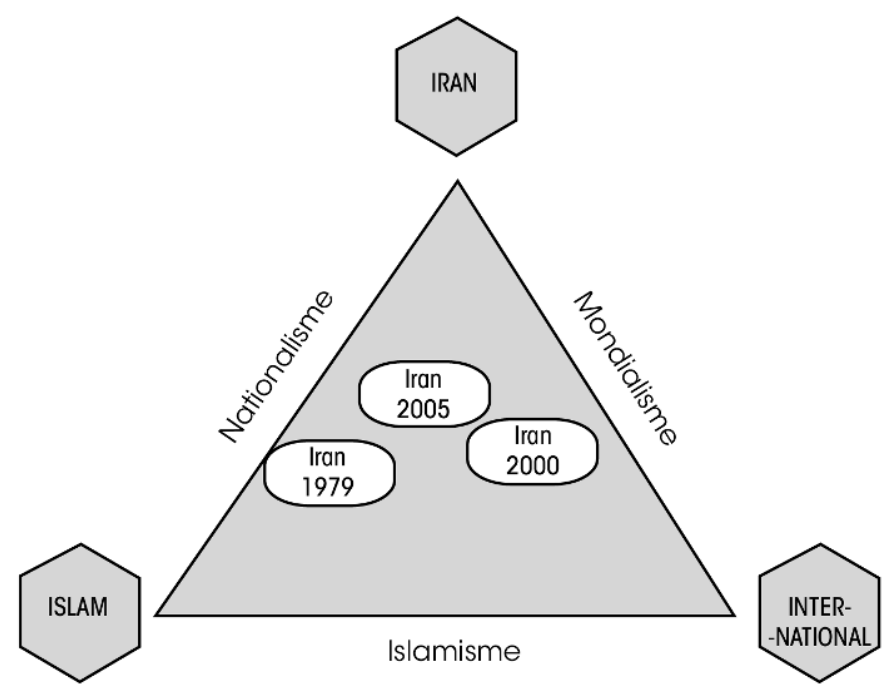

La position de chaque ovale dans le triangle marque sa place par rapport aux trois pôles que sont Islam, Iran et International. 1979 : Khomeiny, 2000 : Khatami, 2005 : Ahmadinejad.

7 Au cours du $\mathrm{xx}^{\mathrm{e}}$ siècle, chacune de ces forces a dominé tour à tour le pays, sans parvenir à un équilibre durable. À l'époque de Réza chah Pahlavi (1923-1941), le facteur «Iran » a dominé. Le nationalisme, la construction de l'État moderne et les contradictions ethniques internes au pays ont marginalisé l'islam et handicapé une ouverture internationale pourtant spectaculaire dans les domaines économiques et éducatifs. Sous le règne de son fils Mohammad Réza (1941-1979), la priorité à l'intégration internationale et la force de la relation avec les États-Unis ont affaibli l'indépendance nationale malgré le discours nationaliste dominant, tandis que l'islam restait marginalisé. L'instauration de la République islamique par l'ayatollah Khomeyni en 1979 a placé l'islam au sommet du pouvoir, sans pour autant occulter les autres composantes de l'identité iranienne : la guerre Irak-Iran (1980-1988) a affirmé de façon dramatique mais bien réelle la vitalité du nationalisme des forces cléricales au pouvoir, tandis que les cultures populaires étaient à la fois valorisées (ainsi des langues locales reconnues par l'article 15 de la Constitution) et combattues (répression contre les kurdes). Quant à l'ouverture internationale, elle a été favorisée par la mondialisation, mais aussi par une politique d'éducation généralisée et l'affirmation d'ambitions scientifiques et technologiques - la politique nucléaire en est l'exemple le plus évident -, tandis que l'islamisme et le nationalisme isolaient le pays aussi bien sur le plan politique que commercial.

Une des ambitions du gouvernement de Mohammad Khatami (1997-2005) a été de chercher un équilibre vertueux entre ces trois forces religieuse, nationale et scientifique/universelle, dont aucune ne saurait être réduite à néant. La volonté illusoire d'éradiquer ou de marginaliser une des composantes de cette identité sans 
prendre la mesure des conséquences sur les autres facteurs peut également expliquer les blocages et les impasses qu'a rencontrés la communauté internationale envers l'Iran. Ainsi, en diabolisant « l'Iran des mollahs », les néo-conservateurs américains ont affaibli les réformateurs islamiques et facilité de fait l'élection du conservateur Mahmoud Ahmadinejad. Dans la crise du nucléaire, une analyse approfondie montre, au-delà des apparences, que les prétentions nationalistes ou islamistes iraniennes sont peut-être marginales comparées aux ambitions des scientifiques et des intellectuels, souvent opposés au régime islamique, c'est-à-dire de la partie « internationale » de la société iranienne qui veut participer sans tarder aux enjeux scientifiques et technologiques du xxI siècle. La compréhension de l'Iran passe par une évaluation constante des proportions d'islam, d'Iran et d'inter-national qui structurent l'Iran actuel et par l'étude de la manière dont ces trois forces interagissent.

\section{L'islam iranien entre Réforme, conservatisme et pragmatisme}

9 Le chiisme demeure le cadre socio-culturel dominant en profondeur la société iranienne. C'est surtout un système d'influence et de relation en raison du lien qui existe entre tout musulman chiite et son marjah, son guide spirituel choisi parmi les grands ayatollahs. Le plus important est peut-être de constater que trois décennies de République islamique ont bouleversé le chiisme iranien et que l'islamologie classique ne peut appréhender qu'une partie du problème ${ }^{6}$.

Le clergé est désormais divisé entre ceux qui participent directement à la gestion de l'État, en suivant fidèlement la doctrine du « velayat-e faqih » (gouvernement du Guide : Rouhollah Khomeyni puis Ali Khamene'i), et ceux qui, tout en affichant une fidélité à un système qui leur donne bien des avantages, privilégient un islam traditionnel et la relation avec les écoles de théologie et les simples fidèles. On peut ainsi distinguer les «mollahs de Téhéran» de ceux de Qom; parmi ces derniers, quelques-uns regardent maintenant vers Najaf, en Irak, qui pourrait redevenir le centre du chiisme mondial.

11 L'expérience de la gestion de l'État, sans interruption depuis 1979, a permis à certains membres du clergé iranien de devenir des experts en politique et en gestion de l'économie. Le plus connu est Ali-Akbar Hashemi-Rafsanjani, qui reste une des personnalités les plus puissantes du pays, mais dont l'échec aux élections présidentielles de 2005 symbolise la fin d'une époque, la fin de « l'Iran des mollahs ».

D'autres religieux ont mis à profit ces expériences en grandeur nature de l'islam politique pour proposer une rupture idéologique et de nouvelles idées en matière religieuse. Ils proposent une forme de laïcité, et une relecture radicale des pratiques islamiques qu'ils décrivent comme héritées de siècles de relations trop étroites entre l'islam et les despotismes et les conservatismes sociaux. Ils invitent à une lecture du Coran dans le contexte d'une société libre. Les questions sur la liberté religieuse et le droit de se convertir à d'autres religions, notamment le christianisme, sont ouvertement abordées, de même que les relations avec les sunnites qui forment la première minorité religieuse du pays $(10 \%$ de la population), mais ne sont pas reconnus comme tels par la Constitution, contrairement aux quelques dizaines de milliers de juifs, chrétiens et zoroastriens. L'expérience iranienne conduit ces religieux à demander une laïcité, une séparation de la religion et de la gestion politique. 
13 Ces nouveaux théologiens réformateurs comme Mohsen Kadivar ou Mojtahed Shabestari s'opposent donc à la fois à ceux qui gèrent l'État et aux religieux traditionnels de Qom. Presque tous sont ou ont été emprisonnés, mais leur audience n'en est que plus grande parmi la population comme parmi les étudiants en théologie de Qom. La victoire d'une Réforme en islam conduite par ces théologiens chiites est encore éloignée, même si leur audience dépasse les frontières du chiisme et s'étend au monde sunnite et au monde arabe ${ }^{\text {? }}$.

14 Le tableau de l'islam iranien serait incomplet sans l'Astaneh Qods, la fondation religieuse qui gère le mausolée de l'imam Réza à Mashhad, le premier lieu de pèlerinage du monde musulman avec plus de 10 millions de pèlerins ${ }^{8}$. Cette fondation, dont les origines remontent au $\mathrm{Xv}^{\mathrm{e}}$ siècle, administre depuis lors les donations des fidèles et est devenue la seconde puissance économique du pays après l'État iranien. Son président, l'ayatollah Vaezi Tabassi, est la seule personnalité nommée par l'ayatollah Khomeyni qui soit toujours en fonction. Le fondateur de la République islamique avait très justement déclaré que cette fondation était le "centre de l'Iran », une réalité mal connue, mais qui demeure.

15 La population des fidèles a été la première bouleversée par ces révolutions religieuses. Une partie des Iraniens rejette une religion qui se sert de l'État pour s'imposer. Malgré leur dynamisme et leur capacité médiatique, il ne faut pas surévaluer le nombre de ces libres-penseurs proches du clergé réformateur et qui forment la base d'une laïcité qui rendrait sa liberté à l'islam comme au politique. Une autre partie de la population, attachée à l'islam chiite, soutient activement la République islamique, mais affirme son autonomie face à un clergé qui est respecté sur le plan religieux, mais pas pour sa gestion politique. Parmi ces nouveaux islamistes, on compte une partie des Gardiens de la Révolution (Pasdaran) et les milices des Basijis (Volontaires).

16 Ces jeunes militants qui avaient 20 ans lors de la Révolution ont été immédiatement engloutis dans la guerre contre l'Irak qui les a écartés de la vie politique du pays pendant huit longues années, laissant le clergé gérer le pays. Après la guerre, ils ont été démobilisés, sauf une minorité de hauts gradés, ils ont entrepris ou repris des études tout en occupant des postes secondaires dans les administrations et en entretenant leur réseau politique et idéologique. Ces générations de la guerre, âgées de 40 à 55 ans, accèdent aujourd'hui au pouvoir et succèdent à celles qui ont fait la Révolution, dominées par le clergé et âgées de plus de 65 ans. L'élection à la présidence de Mahmoud Ahmadinejad en juin 2005 consacre ce changement de génération et surtout l'émergence de nouveaux cadres islamistes non-membres du clergé et parfois même très opposés à certains religieux qu'ils accusent de corruption et d'avoir confisqué la Révolution à leur profit 9 .

17 Les observateurs pensaient que l'arrivée au pouvoir des Pasdaran se ferait plus tard, mais le Guide Ali Khamene'i a été fin politique en lançant dans la bataille électorale des élections présidentielles quatre anciens Gardiens de la Révolution : Mohsen Reza'i, Ali Larijani, Bagher Qalibaf et Mahmoud Ahmadinejad, unis par un passé commun, mais ayant des idées et des méthodes bien différentes. Par cette diversité et l'accession au pouvoir de personnalités plus jeunes, ne portant pas turban, mais légitimées par une guerre qui fut idéologique mais aussi nationale, l'avenir politique de la république islamique reste donc sous contrôle islamique pour au moins quatre années. 


\section{Le nationalisme iranien : les nouvelles identités d'une république}

18 L'Iran de ce début $\mathrm{du} \mathrm{xxI}^{\mathrm{e}}$ siècle reste fidèle à ses riches traditions millénaires. Cependant, les caractères, les capacités et les ambitions des Iraniens ont peu de rapport avec leur héritage ni avec l'idée que l'on se fait généralement de ce pays quand on fonde son analyse sur les images de l'Iran des années 1960 ou des débuts de la Révolution islamique.

La première évidence est le fait que la majorité des Iraniens est née après l'avènement de la République islamique ou a grandi sous ce régime; le régime impérial n'est plus qu'une idée et non un souvenir ou une expérience, si bien que même parmi la nombreuse diaspora iranienne, le projet d'une restauration monarchique est de plus en plus contesté. Le débat politique, porté par les partisans du gouvernement de Téhéran ou par ses opposants, implique de moins en moins l'opposition en exil. Il est animé presque exclusivement au sein du territoire iranien, où la vie politique et sociale est d'une grande diversité et d'une grande vivacité en dépit des limites imposées par l'État islamique. Tous les acteurs de la vie politique iranienne sont désormais en Iran, même ceux d'opposition, malgré l'absence de vrais partis politiques.

Officiellement, plusieurs centaines de "partis " ont été déclarés au ministère de l'Intérieur, mais aucun n'est suffisamment organisé sur tout le territoire et donc capable de gérer un processus électoral. Le parti Mosharekat (Participation), qui soutenait la politique de réforme de Mohammad Khatami est le mieux structuré, mais les divisions internes et les échecs électoraux bloquent l'émergence de nouveaux leaders. Des tentatives de regroupement entre forces "modérées » se multiplient, en particulier à l'initiative de Mehdi Karroubi, ancien président du Parlement réformateur, mais cela ne change pas le paysage politique, car la plupart des leaders restent des « has been » usés par près de trois décennies de gouvernement.

21 L'islam politique semble donc avoir été capable de s'adapter pour conserver le pouvoir, mais le débat politique reste ouvert entre les diverses tendances issues des Pasdaran qui vont de l'étatisme autoritaire au libéralisme et à l'ouverture internationale. L'arrivée au sommet du pouvoir des anciens Gardiens de la Révolution (Pasdaran) traduit plus l'émergence d'une nouvelle génération que la prise en main du pays par un groupe politique. En effet, leur diversité, nourrie par des divergences et des oppositions parfois très fortes, laisse la place à des ouvertures et à des recompositions politiques. Le pragmatisme reste encore une issue. On voit ainsi se dessiner un certain rapprochement entre le nouveau maire de Téhéran Mohammad-Bagher Qalibaf et les partisans de Akli-Akbar Hashemi-Rafsandjani, personnalité soutenue par ses réseaux économiques et clandestins qui lui assurent une pérennité et une adaptabilité incontestées.

Contrairement à une affirmation souvent avancée, l'Iran n'est pas - n'est plus - un pays jeune, surtout en comparaison avec les États voisins. Certes l'âge médian est de 21 ans, mais en raison de la chute de la fécondité depuis 1986 - la plus forte et rapide de l'histoire de l'humanité - ( 2 enfants par femme en moyenne contre 6,8 en 1986) ${ }^{10}$, les nouvelles générations sont beaucoup moins nombreuses, donnant une place dominante aux générations pléthoriques nées entre 1970 et 1985. L'Iran n'est pas un pays de jeunes, mais une nation de jeunes adultes profondément marqués et unis par la très 
dure expérience commune d'une histoire qui a changé leur vie, qu'ils aient soutenu, subi ou combattu le gouvernement et le système islamique imposé à tous. Par leur nombre et leur histoire, ces nouvelles générations qui ont aujourd'hui entre 30 et 50 ans dominent le pays et sont en train d'en prendre le contrôle par le bas.

Le changement le plus important concerne cependant la population féminine. L'abolition du Code de la famille en 1979 et l'obligation faite aux femmes d'adopter les coutumes islamistes, en particulier le port du voile, ont justement attiré l'attention sur la contradiction qui existe entre ces règles imposées et la très forte dynamique de socialisation marquée par la scolarisation massive et la chute généralisée de la fécondité même en zone rurale. Le taux d'alphabétisation des femmes en milieu rural est ainsi passé de 17 \% en 1976 à 62 \% en 1996, tandis que depuis 1998, les femmes sont majoritaires à l'université ${ }^{11}$.

Dans le contexte politique et culturel iranien, nul n'est capable pour le moment de prendre la mesure de l'impact de ces transformations profondes et très rapides de la société féminine de ce vieux pays islamique. Le nombre de femmes qui travaillent, longtemps faible (12\%), augmente très vite, tandis que les «Organisations non gouvernementales » animées par des femmes ou s'occupant de questions liées à leur socialisation sont innombrables et de plus en plus puissantes au niveau politique.

Les revendications ethno-culturelles sont anciennes et fortes dans ce pays où plus de la moitié des habitants n'ont pas le persan pour langue maternelle ${ }^{12}$, mais la priorité n'est plus la tentation irrédentiste, comme ce fut le cas après la Seconde Guerre mondiale en Azerbaïdjan et au Kurdistan. Les revendications portent surtout sur une meilleure insertion des diverses provinces dans les politiques de développement et sur la prise en compte des diversités culturelles et surtout religieuses par l'État central chiite. La première minorité religieuse en Iran est celle des sunnites (10\% de la population) qui est assimilée aux « musulmans » et n'est pas reconnue comme telle, contrairement aux chrétiens, zoroastriens et juifs qui ont chacun un député et des lieux de culte. La question ethno-culturelle est une réalité incontournable dans les provinces concernées, mais c'est dans les grandes villes, où vivent désormais des migrants venus de tout le pays, que se créent de nouveaux rapports de force. Il n'y a pas de mosquée sunnite à Téhéran. Les progrès de l'éducation, l'urbanisation et la construction d'une nouvelle classe moyenne ont transformé les conditions de l'expression des diversités culturelles qui ne sont plus le propre des provinces pauvres ${ }^{13}$.

26 Le nationalisme iranien reste fondé sur le sentiment obsidional d'être une île encerclée de forces hostiles, mais une dynamique nouvelle est également nourrie par le sentiment que ce pays a d'immenses potentialités et qu'il est en mesure de jouer un rôle international de premier plan. Cette conviction nationaliste nouvelle est alimentée par la différence croissante entre le niveau d'éducation et d'information de la population, notamment des nouvelles générations de jeunes adultes, et la médiocrité économique et l'isolement culturel de la grande majorité des habitants, dans les provinces. L'élection surprise de M. Ahmadinejad est en partie l'expression de ces contradictions. Les revendications sont dirigées contre un système gouvernemental contesté, mais aussi contre une communauté internationale qui semble ne pas voir les changements et ne regarde que les élites au pouvoir. La crise du nucléaire, qui fait l'unanimité du pays en faveur non de l'arme atomique, mais de l'accès sans contrainte à la recherche nucléaire, est devenue le symbole de ce nouveau nationalisme complexe 
dont l'islamisme ne constitue qu'un aspect, peut être surdimensionné par la communauté internationale.

\section{L'inexpérience internationale des nouvelles générations}

Depuis sa création, la République islamique n'a jamais cessé d'affirmer son hostilité à l'égard d'un grand nombre d'États, particulièrement les États-Unis et Israël, ni sa méfiance envers les institutions internationales et les règles économiques, politiques et culturelles acceptées par la communauté des nations. Cette attitude allant de pair avec un souci légaliste, parfois obséquieux, de respect des formes et des lois internationales. Au-delà des outrances liées au contexte révolutionnaire puis à la guerre Irak-Iran, cette volonté d'affirmer son identité, cette crainte d'être entouré de forces hostiles et le refus de collaborer de façon sereine procèdent d'une attitude traditionnelle à la fois obsidionale et nationaliste, poussée à son paroxysme. L'Iran est plus que jamais une île, sûre de sa supériorité et de son bon droit, mais qui commence à prendre la mesure des réalités, notamment dans les domaines scientifiques, technologiques et économiques.

Les limites de cette autarcie - de cet autisme - commencent à être visibles. La société iranienne et même certains responsables politiques ont une bonne connaissance de l'évolution du monde depuis un quart de siècle et prennent la mesure du retard pris par l'Iran islamique du fait des contraintes imposées notamment par l'embargo américain ${ }^{14}$, mais surtout à cause de l'auto-embargo que le pays s'inflige en ne faisant rien pour intégrer la communauté internationale. L'annonce des succès technologiques iraniens dans l'industrie nucléaire formulée le 12 avril 2006 ne saurait faire illusion. La réussite scientifique et technologique pour mettre en œuvre, après dix ans de travail, les matériels et techniques importés clandestinement du Pakistan dans le cadre du réseau d'Abdul Qadeer Khan, ne peut faire oublier que l'industrie iranienne n'est pas capable d'exporter le moindre produit industriel ni le moindre service.

En imposant de fait un monopole d'État dans presque tous les domaines, la Constitution iranienne (article 44) ${ }^{15}$ place le secteur privé, et surtout les entreprises étrangères, en situation de précarité permanente malgré la politique de privatisation accélérée depuis 1990. Il a fallu attendre 2002 pour que le Parlement vote une loi sur la protection des capitaux étrangers. La France a été le premier pays à ratifier un accord bilatéral dans ce cadre, mais les investissements étrangers restent insignifiants, bien qu'ils soient indispensables pour satisfaire les besoins élémentaires du pays et assurer des emplois adaptés aux nouvelles générations désormais bien formées. Le premier investissement étranger significatif depuis 1978 est celui de Renault qui a créé en 2003 une «Joint venture ", tandis que les compagnies pétrolières étrangères passent des contrats de " buy back » inadaptés à des politiques industrielles à long terme. Malgré les discours, le système politique iranien ne fait rien ou trop peu, pour établir la confiance indispensable aux relations économiques durables et à l'échelle des besoins du pays. Alors que l'immense majorité des Iraniens fait preuve d'une xénophilie d'autant plus réelle qu'elle souhaite parti-ciper à la vie $\mathrm{du} \mathrm{xxI}^{\mathrm{e}}$ siècle, le contexte économique et culturel iranien reste non pas hostile, mais craintif envers tout ce qui vient de l'étranger. 
30 La plupart des anciens Gardiens de la Révolution qui accèdent depuis peu à des responsabilités de haut niveau n'ont en effet aucune pratique du monde qui les entoure. Plus grave encore, leur seule expérience a été celle de la guerre Irak-Iran. L'isolement du pays depuis vingt-cinq ans et la politique officielle de «lutte contre l'agression culturelle occidentale » ne leur a pas permis d'aller à l'étranger si bien qu'ils ne parlent pas ou peu les langues étrangères et surtout qu'ils n'ont pas d'expé-rience personnelle des cultures du monde. On est loin des conseils donnés aux premiers étudiants boursiers iraniens se rendant en Europe en 1927 à qui on demandait de « devenir français » tout en leur apprenant à nouer une cravate...

31 La politique de "dialogue des civilisations» mise en œuvre par le président Mohammad Khatami à partir de 1997 a permis une ouverture incontestable, mais rapidement bloquée et limitée à un réseau d'intellectuels. Parmi les personnalités islamistes, et notamment parmi les anciens Gardiens de la Révolution, il existe néanmoins une élite qui a résidé à l'étranger et a acquis une connaissance approfondie des cultures et des dynamiques contemporaines. Cette nouvelle élite à la fois islamiste, nationaliste et internationaliste est cependant peu nombreuse en comparaison des nombreux Iraniens de la même génération qui n'ont pas servi le régime islamique et n'ont jamais cessé de vivre avec leur siècle. Une alliance entre ces deux élites «internationales» est possible, mais pour l'heure, le conflit culturel est tel sur cette question de l'ouverture internationale que les jeunes iraniens diplômés quittent le pays en masse. Le chiffre de 200000 par an avancé en 2002 par le ministère de l'Enseignement supérieur ${ }^{16}$ semble exagéré, mais la réalité est incontestable.

La diaspora iranienne formée d'émigrés qui ont quitté le pays au moment de la Révolution, résidant surtout aux États-Unis et n'ayant plus de liens étroits avec leur pays d'origine, est devenue plus complexe. La seconde génération des exilés atteint l'âge adulte et cherche à découvrir le pays de ses parents, tandis qu'arrive une nouvelle vague d'émigrés composée surtout des jeunes Iraniens qui quittent le pays pour étudier ou ne pas perdre leur jeunesse dans l'austérité islamique, comme leurs parents. L'intégration de cette communauté hétérogène dans la vie politique iranienne semble illusoire ou au moins marginale.

En effet, pour des raisons financières et sociales évidentes, l'immense majorité des jeunes iraniens restent en Iran. Là, ils n'ont presque aucune possibilité de rencontrer des étrangers ou d'avoir une expérience personnelle des différentes cultures autre que de façon virtuelle par Internet, la télévision, les vidéos, le téléphone ou les livres. L'isolement de l'Iran a pour conséquence l'extrême rareté des étrangers qui vivent de façon permanente dans le pays. Au milieu des 70 millions d'Iraniens vivent moins de 4000 étrangers expatriés, résidents permanents ${ }^{17}$. Quant au tourisme, malgré une politique touristique affichée, il n'attire guère que 200000 personnes qui ne fréquentent que les grands sites archéologiques... Si Téhéran était une capitale internationale accueillant 100000 étrangers, comme Istanbul, New Delhi ou Le Caire, les Iraniens auraient des opportunités de connaître le monde actuel, mais le système politico-culturel islamique serait mis au défi par une telle mixité souhaitée par l'immense majorité des Iraniens.

L'évolution de l'Iran semble donc très différente de celle de la Chine, même si, dans les deux cas, on constate une volonté de fermeture aux cultures internationales et à l'universalité des droits de l'Homme. Une des spécificités de l'Iran est l'étonnante différence que l'on constate entre le haut niveau de connaissance théorique que la 
majorité de la population a du monde actuel, en particulier sur le plan scientifique, et la faiblesse de l'expérience vécue. Cette situation explique en partie pourquoi les composantes nationaliste et islamique du gouvernement sont aujourd'hui renforcées, par crainte de ne pas pouvoir gérer convenablement un univers mal connu alors que l'attraction du pôle international est plus fort que jamais dans la société.

En octobre 2003, l'Iran a été confronté - par choix ou par nécessité - à une orientation stratégique qui semble irréversible. En effet, en l'espace de deux semaines se sont succédé l'attribution du prix Nobel de la paix à Chirine Ebadi, fort signal international appelant au respect des droits de l'Homme; l'acceptation par l'Iran des propositions européennes sur le moratoire nucléaire et sur un processus d'intégration économique, politique et de sécurité; et enfin l'accord industriel entre Renault et le ministère iranien de l'Industrie ${ }^{18}$. Ces trois événements, qui concernent respectivement les domaines islamique, national et international, marquent une rupture avec le passé et la prise en compte des dynamiques réelles du pays. La mise en œuvre de ces nouvelles orientations ne se fait pas sans à-coups, comme le montre la crise du nucléaire, mais l'hypothèse d'une évolution positive reste réaliste, car les nouvelles générations qui accèdent aux affaires, y compris les anciens Gardiens de la Révolution, ont acquis une formation scientifique honorable et intègrent les dynamiques internationales bien plus que les religieux qui gèrent le pays depuis 1979. L'inexpérience internationale de ces nouvelles élites pose problème, mais elle n'est pas sans solution.

Dans ce contexte, la crise du nucléaire et la politique iranienne en Irak confirment de façon paradoxale la montée en puissance de la composante internationale de l'identité iranienne nouvelle, même si le discours islamiste et nationaliste domine. Un échec sur la question nucléaire provoquerait une crise dramatique, mais la gravité de la situation peut également conduire au renforcement de l'insertion internationale de l'ancienne Perse enfin reconnue comme une puissance du xxI ${ }^{e}$ siècle. Le fait que pour la première fois depuis 1979, l'administration américaine ait autorisé un ambassadeur à rencontrer son homologue iranien et que l'Iran ait accepté l'invitation le 16 mars 2006, est peutêtre le signe d'un changement durable, malgré le manque d'expérience de cette nouvelle puissance régionale qui n'est plus un empire et n'a pas encore trouvé son équilibre comme république.

\section{NOTES}

1. J.-P. Digard, B. Hourcade et Y. Richard, L'Iran au XXe siècle, Paris, Fayard, 1996.

2. F. Khosrokhavar et $\mathrm{P}$.Vieille, Le discours populaire de la révolution iranienne, Paris, Contemporanéité, 1990 ; F. Adelkhah, La Révolution sous le voile, Paris, Karthala, 1991.

3. La division population de l'ONU a longtemps refusé de reconnaitre la réalité de la chute de la fécondité en Iran depuis 1986, arguant que l'idéologie de la République islamique était nataliste.

4. L'accord politique et militaire signé en 1962 entre l'Iran et les États-Unis pour faire front à l'URSS a été accompagné d'une politique de réformes dynamiques, contestées, mais qui ont mis l'Iran sur la voie du développement avec des mesures comme la réforme agraire, le droit de vote 
pour les femmes et une politique volontariste en matière d'éducation, de santé, d'infrastructures et d'industrialisation.

5. Sur l'histoire récente de l'Iran : J.-P. Digard, B. Hourcade et Y. Richard, op. cit.

6. Sur les dynamiques actuelles du chiisme iranien : Yann Richard, L'islam chiite, Paris, Fayard, 1991 et Olivier Roy, L'échec de l'islam politique, Paris, Seuil, 1995.

7. Roxanne D. Marcotte, « Religion and Freedom : Typology of an Iranian Discussion », Australian Religion Studies Review, 18, 1, 2005, pp. 49-67.

8. Malgré le nombre relativement faible de chiites et la quasi-absence de pèlerins venant d'Irak, l'importance de cette ville sainte vient du fait que le pèlerinage se fait toute l'année et qu'elle accueille de plus en plus de musulmans, même sunnites, venant d'Asie centrale.

9. Sur les changements politiques récents en Iran : B. Hourcade, "Iran : retour à l'islamisme?", Politique internationale, 109, 2005, pp. 85-200, et le volume spécial de Géoéconomie, 36, 2005 dirigé par M. Makinsky : Iran, l'heure des ruptures?

10. Cette révolution démographique a été mise en évidence pour la première fois par Marie Ladier-Fouladi, Population et politique en Iran, Paris, Institut national d'études démographiques, 2003.

11. Ces changements profonds de la société féminine iranienne ont été analysés en détail grâce à un programme de recherche réalisé en 2002 sous la direction de Azadeh Kian-Thiébaut et Marie Ladier-Fouladi, dans la cadre d'une collaboration entre l'équipe de recherche CNRS Monde iranien, l'Institut français de recherche en Iran et le Centre de statistiques d'Iran. Les principaux résultats de cette enquête nationale réalisée auprès de près de 50000 personnes dans tout le pays sont publiés sur http//:www.ivry.cnrs.fr/iran. A. Kian-Thiébaut, «From Motherhood to equal Rights Advocates : The Weakening of Patriarchal Order ", Iranian Studies, 38, 1, 2005, pp. 45-66.

12. La langue nationale est comprise par plus de $90 \%$ de la population du fait des progrès de la scolarisation, mais les langues locales sont encore très pratiquées, notamment dans certaines provinces, comme le Baloutchistan ou le Kurdistan et surtout l'Azerbaïdjan, où les identités régionales restent vivantes et fortes.

13. B. Hourcade, «Le fait ethnique en Iran: risque de conflit ou enjeu dépassé par l'urbanisation?", Géoéconomie, 36, 2005, pp. 85-96.

14. L'embargo commercial des États-Unis constitue un handicap tous les jours plus grand pour les entreprises non américaines travaillant en Iran qui ont besoin de brevets ou de pièces venant d'Outre-Atlantique. La loi d'Amato qui « interdit », en contradiction avec les règles de l'OMC, les investissements pétroliers en Iran reste une menace pour les rares entreprises qui travaillent dans ce pays. Le groupe Total est le premier à être passé outre dès 1995.

15. Une traduction en français de la Constitution révisée en 1989 a été établie par M. Potocki, Constitution de la République islamique d'Iran 1979-1989, Paris, l'Harmattan, 2004.

16. Cité par M.-R. Djalili, Iran : l'illusion réformiste, Bruxelles, Complexe, 2004.

17. Non compris les travailleurs immigrés afghans et ceux vivant en célibataire dans des chantiers de travaux publics ou pétroliers.

18. B. Hourcade, « Le réveil de l'Iran », Le Monde diplomatique, février 2004. 


\section{RÉSUMÉS}

La Perse était au début du $\mathrm{XX}^{\mathrm{e}}$ siècle un pays faible et marginal. Un siècle plus tard, l'Iran est devenu une puissance régionale bien réelle, quoique contestée. Bien des analyses ne prennent pas en compte ces transformations rapides, inachevées mais profondes, en confinant le pays dans son lointain passé prestigieux. L'identité iranienne d'aujourd'hui est le résultat d'un équilibre difficile entre trois forces : l'islam, l'Iran et l'international. Un des problèmes majeurs du pays, isolé depuis un quart de siècle, est son inexpérience des réalités internationales, ce qui génère des crises incessantes surtout avec les nouvelles générations issues de la guerre Irak-Iran. l'Iran n'est plus un empire. Il cherche à être reconnu comme une nouvelle puissance régionale durable.

In 1900, Persia was a weak and marginal state. A century later, Iran has become a true but nevertheless contested regional power. Numerous studies do not take into account these recent rapid, unfinished but deep transformations, and confine the country to its prestigious ancient history. Current Iranian identity is the result of a difficult balance between three different forces: Islam, Iran and the International. Following twenty-five years of isolation, lack of international experience is one of the major problems faced by Iran. This gives rise to perpetual crises, in particular where the generations who grew up during the Iraq-Iran war are concerned. Iran is no longer an empire and wants to be seen as a sustainable new regional power.

\section{INDEX}

Mots-clés : nationalisme, islamisme, politique internationale

Keywords : Nationalism, islamism, international relations

Index géographique : Iran

\section{AUTEUR}

\section{BERNARD HOURCADE}

Bernard Hourcade, directeur de recherche au CNRS, est géographe. Il a dirigé l'Institut français de recherche en Iran (1978-1993) puis l'UMR Monde iranien (1993-2005). Ses travaux ont d'abord porté sur le développement rural dans l'Alborz, puis sur la géographie sociale et politique de l'Iran, notamment à partir de l'analyse du développement des villes. Principales publications sur l'Iran : L'Iran au $\mathrm{Xx}^{\mathrm{e}}$ siècle, (coll.) Paris, Fayard, 1998 ; Iran, les identités -nouvelles d'une république, Paris, Belin, 2003, Atlas de Téhéran métropole, Téhéran, 2005.

hourcade@ivry.cnrs.fr 